\title{
A HIRES/KECK SPECTROSCOPIC INVESTIGATION OF THE MEASUREMENT OF SODIUM IN THE ATMOSPHERE OF HD 209458b
}

\section{Citation}

Langland-Shula, Laura E., Steven S. Vogt, David Charbonneau, Paul Butler, and Geoff Marcy. 2009. "A HIRES/KECK SPECTROSCOPIC INVESTIGATION OF THE MEASUREMENT OF SODIUM IN THE ATMOSPHERE OF HD 209458b." The Astrophysical Journal 696 (2): 1355-66. https:// doi.org/10.1088/0004-637x/696/2/1355.

\section{Permanent link}

http://nrs.harvard.edu/urn-3:HUL.InstRepos:41397471

\section{Terms of Use}

This article was downloaded from Harvard University's DASH repository, and is made available under the terms and conditions applicable to Other Posted Material, as set forth at http:// nrs.harvard.edu/urn-3:HUL.InstRepos:dash.current.terms-of-use\#LAA

\section{Share Your Story}

The Harvard community has made this article openly available.

Please share how this access benefits you. Submit a story.

\section{Accessibility}




\title{
A HIRES/KECK SPECTROSCOPIC INVESTIGATION OF THE MEASUREMENT OF SODIUM IN THE ATMOSPHERE OF HD 209458b*
}

\author{
Laura E. Langland-Shula ${ }^{1}$, Steven S. Vogt ${ }^{1}$, David Charbonneau ${ }^{2,5}$, Paul Butler $^{3}$, And Geoff Marcy ${ }^{4}$ \\ ${ }^{1}$ UCO/Lick Observatory, University of California, Santa Cruz, CA 95064, USA \\ ${ }^{2}$ Harvard-Smithsonian Center for Astrophysics, 60 Garden Street, Cambridge, MA 02138, USA \\ ${ }^{3}$ Department of Terrestrial Magnetism, Carnegie Institute of Washington, Washington, DC 20015-1305, USA \\ ${ }^{4}$ Department of Astronomy, University of California, Berkeley, CA 94720, USA \\ Received 2008 April 12; accepted 2009 January 14; published 2009 April 24
}

\begin{abstract}
We present high-resolution High Resolution Echelle Spectrometer (HIRES)/Keck spectra of HD 209458, and a Monte Carlo variation on the basic method used by other workers, to look for the excess in-transit absorption in the NaD doublet at $5893 \AA$ due to the extrasolar planet. The HIRES data, binned by bandpass, allow a direct comparison with previous results. We find $>3 \sigma$ results in most test bandpasses around the $\mathrm{NaD}$ doublet, including relative absorption of $(-108.8 \pm 25.7) \times 10^{-5}$ in the "narrow" bandpass used by other workers. This is $\approx 4.7$ times larger than the "narrow" results reported by Charbonneau et al. for HD $209458 \mathrm{~b}$. However, $>2 \sigma$ absorption is detected in some weak $\mathrm{Fe}$ I and $\mathrm{Ni}$ I lines that were tested for comparison, raising concern about the uncertainties introduced by continuum-fitting and terrestrial atmosphere subtraction.
\end{abstract}

Key words: planetary systems - stars: atmospheres - stars: individual (HD 209458) - techniques: photometric

\section{INTRODUCTION}

Ever since the discoveries of the first extrasolar planets orbiting main-sequence stars (Mayor \& Queloz 1995; Marcy \& Butler 1998), scientists and laypeople alike have been excited by the possibility of finding Earth-like planets that display chemical signs of habitability. While actual discovery of such Earth-like planets lies years into the future, astronomers are currently developing techniques to determine the compositions of some extrasolar giant planets. The techniques currently being used by several groups, in addition to laying the foundations for future analysis of terrestrial planets, will also provide current researchers with clues about the formation and structure of close-in extrasolar giant planets.

Though close-in extrasolar giant planets (hereafter CEGP; those extrasolar planets with periods less than one to three weeks; Cameron 2002; Ksanfomality 2004, and several others) were unexpected by planet hunters at the time of their discovery, their star-planet systems provide the best opportunities to start to determine the compositions of extrasolar planets. There are three basic strategies for trying to determine composition: using direct imaging when the planet is spatially separated from the star (Davies 1980), using reflected stellar light when the planet is in opposition relative to the Earth and its parent star (Charbonneau 2003), and using transmitted light when a transiting planet is in conjunction relative to the Earth and its parent star (Seager \& Sasselov 2000; Brown 2001; Hubbard et al. 2001).

There have been many attempts to detect a transmitted spectrum from the CEGP orbiting HD 209458 and HD 189733. Brown et al. (2000) discussed theoretical predictions that the depths of absorption features may be as large as $10^{-3}$ of the parent star's continuum intensity. They searched in one visible High Resolution Echelle Spectrometer (HIRES) spectrum and one near infrared NIRSPEC spectrum of HD 209458, but

\footnotetext{
* Based on data obtained with the W. M. Keck Observatory, which is operated by a partnership consisting of the California Institute of Technology, the University of California, and the National Aeronautics and Space Administration.

5 Alfred P. Sloan Research Fellow.
}

reported no detections. Brown et al. (2002) reported an upper limit of the $2.3 \mu \mathrm{m} \mathrm{CO}$ band planet/star ratio which is three times higher than the fiducial models of Brown (2001).

Charbonneau et al. (2002, hereafter C2002) reported $\mathrm{NaD}$ absorption of $(2.32 \pm 0.57) \times 10^{-4}$ in HD 209458. Vidal-Madjar et al. (2003) reported Lyman $\alpha$ absorption of $15 \% \pm 4 \%$ and Vidal-Madjar et al. (2004) reported 2-3 $\sigma$ detections of carbon and oxygen in HD 209458. Narita et al. (2005, hereafter N2005) reported upper limits of a few tenths of a percent for several lines in HD 209458, including $\mathrm{NaD}$ doublet. Other groups have reported recent and significant results. Snellen et al. (2008) (hereafter S2008) reported a ground-based detection of sodium in HD 209458b. Sing et al. (2008) found $\mathrm{Na}$ absorption of $1.1 \times 10^{-3}$ in a $4.4 \AA$ wide band. They also report the presence of an additional absorber at $\approx 6250 \AA$. Redfield et al. (2008) reported the first ground-based detection of an extrasolar planet atmosphere. They reported Na I absorption in HD 189733b of $(-67.2 \pm 20.7) \times 10^{-5}$ in the same "narrow" band as C2002

Because of uncertainty about the fate and future of the Hubble Space Telescope (HST), and until next-generation space telescopes are fully operational, ground-based facilities offer the most immediate opportunities to study the compositions of extrasolar giant planets. In this work, we present another ground-based attempt to detect the planetary atmosphere of HD 209458b. This work concentrates on the sodium resonance doublet at $5893 \AA$, which is predicted to appear in HD $209458 \mathrm{~b}$ (Seager \& Sasselov 2000; Hubbard et al. 2001).

\section{OBSERVATIONS AND DATA REDUCTION}

HD 209458 was the first star known to host a transiting planet (Henry et al. 2000; Charbonneau et al. 2000; Mazeh et al. 2000). It is a G0V star with $V=7.64$, located at a distance of $47.08 \mathrm{pc}$. Its planet has $M \sin (i)$ of $0.66 \pm 0.03 M_{\text {Jup }}$, a radius of $1.32 \pm$ $0.025 R_{\text {Jup }}$ (Knutson et al. 2007), and an inclination of $86.6 \pm$ 0.14 (Brown et al. 2001). The orbit of HD 209458b has a semimajor axis of $0.045 \mathrm{AU}$, a period of $3.524738 \pm 0.000015$ days, and zero eccentricity. On UT 2000 July 16, a full transit of HD 209458 was observed with Keck-I/HIRES (Vogt et al. 1994), 
using the B1 slit $(0.57 \times 3.5$ arcsec $)$ and KV370 filter to allow transmission from 3500 to $6500 \AA$ while blocking unwanted orders from the cross-disperser. At a resolving power $\times$ slit width of 39,000 arcsec (HIRES manual), a resolving power of $\approx 68,400$ is achieved. The weather and seeing were generally excellent. At that time, the telescope could not reliably slew to new targets (although guiding on a given target, i.e., HD 209458, was fine). As a result, fewer calibration sources than desirable were visited.

All the images were read out in the dual readout mode, where (after trimming) row pixels 0:1023 and 1024:2047 are read out by separate electronics, and as a result show different gain and nonlinearity, which are significant at this level of precision. All images were also binned by 2 along the cross-dispersion axis. Since the resulting readout times were approximately $50 \mathrm{~s}$, we were not readout time limited when observing HD 209458 with 240 s exposures. There is one gap of $\approx 15$ minutes in the transit observation time series when we tried unsuccessfully to move to a B star.

\subsection{Preliminary Reduction}

Sets of dark exposures were obtained which correspond to each science exposure time. Visual inspection of the darks at equal exposure times showed that the median value fluctuated significantly from image to image. For each exposure time, a reference image was selected, and the other exposures were scaled by the ratio of the overscan values on the right- and lefthand sides. Comparison of the scaled images for the various darks seems to be in good agreement. The pixel values were converted from raw data numbers to $e^{-}$, assuming a gain of $4.8 e^{-}$per data number, and a nonlinear response function derived from earlier high signal-to-noise ratio (S/N) HIRES observations (Charbonneau et al. 1999). Different values were assumed for the nonlinear response of the left- and righthand readouts (the presumed source of the nonlinearity). The overscan-scaled master darks were subtracted from the HIRES science images.

Cosmic rays were rejected by examining the time series of each individual pixel over the images of the same target. Corrupted values were replaced by values derived from a polynomial fit for that pixel in time. The fraction of pixels replaced was small $\left(\approx 13.5 \times 10^{-4}\right.$ for HD 209458). Some cosmic ray hits remained, and were removed later by examining the ratios of reduced one-dimensional spectra.

The same spectrographic order extraction mask was used for all the images because the image motion in the cross-dispersion direction over the course of the night was less than a pixel. A polynomial $y(x)$ was fit to the bottom echelle order. The other orders were bootstrapped from $y(x)$. The extraction mask was then specified by extending $y(x) \pm 6$ pixels in the crossdispersion direction for each order.

Four sets of continuum lamp exposures were taken throughout the night. Each continuum lamp exposure was scaled-dark subtracted, and corrected for detector gain and nonlinearities. A two-dimensional master flat was constructed for each of the four series, by taking the median value (at each pixel) over all of the renormalized exposures in the series. A small but significant scaling offset in each order (roughly $8 \%$ ) between the left- and right-hand side of the CCD (a gain difference) was evaluated by taking the median of 3 pixels just to the left and right of the center of the chip. Each of the master flats was corrected so that there is no step across the center of the chip, by scaling the left side.
The flats were then multiplied by the order extraction masks, producing "corrector" arrays. The corrector arrays are multiplied with each two-dimensional echelle image to produce onedimensional spectra. Special care was taken with the notorious felt tip pen mark (added by the CCD manufacturer to remind us that this is an engineering-grade device) in the order of 20; those values were set to zero. Rather than combining all the four master correctors, we chose the corrector from the series closest in time to the start of the night (the third series), because the values for a given pixel in the master flat from each series varied significantly. For each science exposure, we multiplied by the corrector and summed across the $y$-direction. The resulting one-dimensional spectra retain the true exposure levels, thus facilitating error estimates.

For all science exposures of HD 209458, we then calculated the HJD for the time at the center of the exposure, the planetary orbit projected forward from the best-fit $\mathrm{C} 2002$ period and phase $\left(P=3.524738\right.$ days and $\left.T_{c}=2451659.93675\right)$, and the transit light curve (assuming no limb darkening) for the purpose of identifying where a given exposure falls during transit. Knutson et al. (2007) also measured a new period for the planet which is consistent with C2002, and a new $T_{c}$ which only differs by $1 \mathrm{~s}$, so uncertainties in the ephemeris are negligible for our purposes.

\subsection{Additional Reduction}

A few more reduction steps were necessary before the data could be in a form usable for differential spectroscopy: continuum-normalizing the echelle blaze shape, removing as many of the terrestrial atmospheric lines as possible, crosscorrelating the spectra at the subpixel level to remove horizontal Doppler shifts due to relative motion between the Earth and the star, and removing cosmic rays that survived the previous cosmic-ray correction. If all nonstellar contaminants in the data are removed, most of the remaining variation in the timeseries of an individual pixel is due to the planet's atmosphere, but each of the steps taken to further reduce the data can introduce additional errors that must be understood. In addition, multiple different methods or parameters might be used for each reduction step, so within one reduction step there may be multiple sources of uncertainty depending on which method or parameters are used.

\subsubsection{Continuum Blaze Normalizing}

Spectra gathered with echelle spectrometers suffer from pronounced curvature of the continuum due primarily to the echelle blaze function. Tiny shifts in the incident light path (from atmospheric effects and telescope guiding errors) produce miniscule changes in the light path of each wavelength through the optics, which results in variable vignetting along the spectrometer's optical path and introduce spatial variations in the continuum beyond the simple throughput curve. The shape of the continuum blaze function varies subtly and unpredictably from exposure to exposure, even after dividing by a continuum arc lamp, by amounts larger than the level of precision required.

The remaining echelle blaze function in the continuum is removed using the following procedure. First, we choose a continuum mask from the exposures of HD 209458 to probe small-scale fluctuations, fit a polynomial to the continuum, and divide each spectrum by the polynomial. Second, we remove terrestrial atmospheric water lines that contaminate the spectrum (discussed in Section 2.2.2). Finally, we do a second iteration of the polynomial continuum fit. 


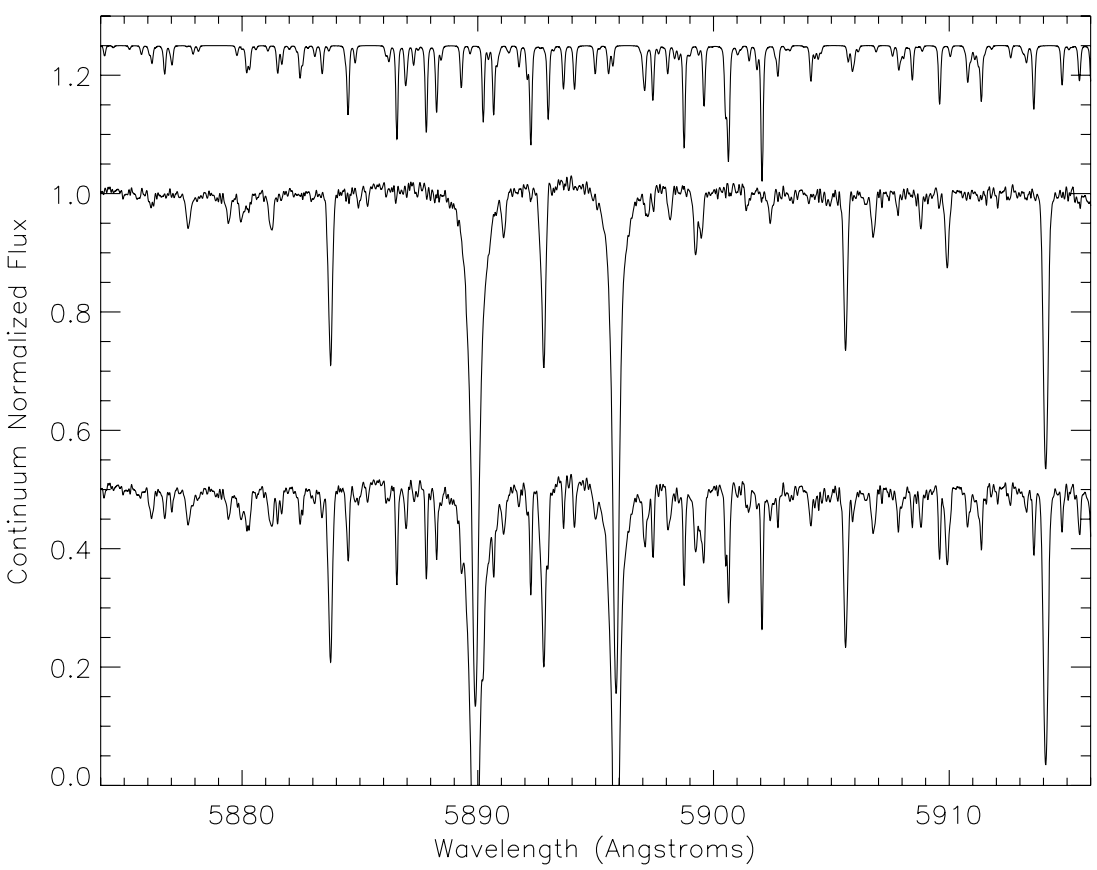

Figure 1. Bottom spectrum is a continuum-normalized sample spectrum of HD 209458 over the blue half of the echelle order containing the NaD lines, offset by -0.5 . The top spectrum is a HITRAN model of atmospheric lines, convolved to the same resolution as the data and offset by 0.25 . The middle spectrum is the difference between the data and the model, an example of removing atmospheric lines from the spectra.

Ideally, this procedure will normalize the continuum to a value of 1 , and differentially preserve the strength of spectral features with respect to the continuum. However, three factors can introduce uncertainty into the results of this process. First, because the echelle blaze function can make the photon count in each continuum pixel vary from exposure to exposure over time, there is no automated way to identify the continuum, so a continuum mask must be selected by hand. There may be weak lines (misidentified as noise) or wings of stronger lines in the regions marked as continuum. Second, the most appropriate degree of polynomial to fit is not known a priori, thus the use of an inappropriate function to normalize the continuum blaze function could introduce broad artificial features that appear to be absorption or emission. Lastly, noise in the continuum may be large enough to detrimentally affect the polynomial fitting.

Due to computational constraints, it is not possible to fit a single polynomial across an entire HIRES order and achieve continuum-fitting residuals of less than $1 \%$. Residuals of $1 \%$ will swamp an in-transit absorption signal which is on the order of one part in $10^{4}-10^{5}$. By splitting the echelle order in half, and fitting a high-order (seventh to ninth degree) polynomial to each half, continuum-fitting residuals can be reduced to about $0.5 \%$. N2005 also found that continuum-fitting errors could be reduced by considering only 1000 pixels around a feature of interest.

\subsubsection{Terrestrial Line Removal and Wavelength Calibration}

Humidity in the Earth's atmosphere produces water features in the spectra. In the echelle order, which contains the $\mathrm{NaD}$ lines, HIRES reveals hundreds of individual water spectral lines. As shown in Figure 1, more than 20 terrestrial atmospheric water lines of various intensities contaminate the 5887-5899 A narrow band defined by $\mathrm{C} 2002$. If not properly removed, variations of the terrestrial water lines with zenith angle can mask variations of the $\mathrm{NaD}$ lines.
Two methods for removing terrestrial lines from the spectra were used. One method was to generate models for the expected transmission profile of the atmosphere for each spectrum individually, and then divide by the models. Another way to remove the atmospheric lines was to use rapidly rotating B stars as references for removing the atmosphere lines.

We used the HITRAN molecular spectroscopic database ${ }^{6}$ to model the atmospheric transmission spectrum with lineby-line resolution. The HITRAN data are easily accessible through a graphical interface called PcLnWin ${ }^{\odot},{ }^{7}$ which allows a user to enter detailed atmospheric and light path geometry parameters, model atmospheric transmittance and radiance at line-by-line spectral resolution, and output ASCII-convertible spectrum files.

The abundance of water in the Earth's atmosphere is the primary contributor to the strength of terrestrial lines at $\mathrm{NaD}$. The climate in the Hawaiian Islands is dominated by the Pacific trade wind, which flows from the northeast to the southwest and must compress when it reaches the Hawaiian Island chain. Although the "lee" sides of the islands have had "drought" conditions for several years (Lawrimore et al. 2000; Levinson \& Waple 2003), the islands still experience precipitation almost every day, a visible harbinger of elevated humidity in the atmosphere. Atmospheric models were constructed from direct comparisons to weather data from the United Kingdom Infra-Red Telescope (hereafter UKIRT) on Mauna Kea, since data from Keck/HIRES was not immediately available (see Figure 2).

The model geometry and atmosphere used in PcLnWin had the following properties: slant path through the atmosphere starting at $4.2 \mathrm{~km}$ (the elevation of Keck Observatory) and

\footnotetext{
6 Developed and maintained by Laurence Rothman at the Atomic and Molecular Physics Division of the Harvard-Smithsonian Center for Astrophysics.

7 A commercial version of the Air Force Phillips Laboratory's FASCOD3P model.
} 


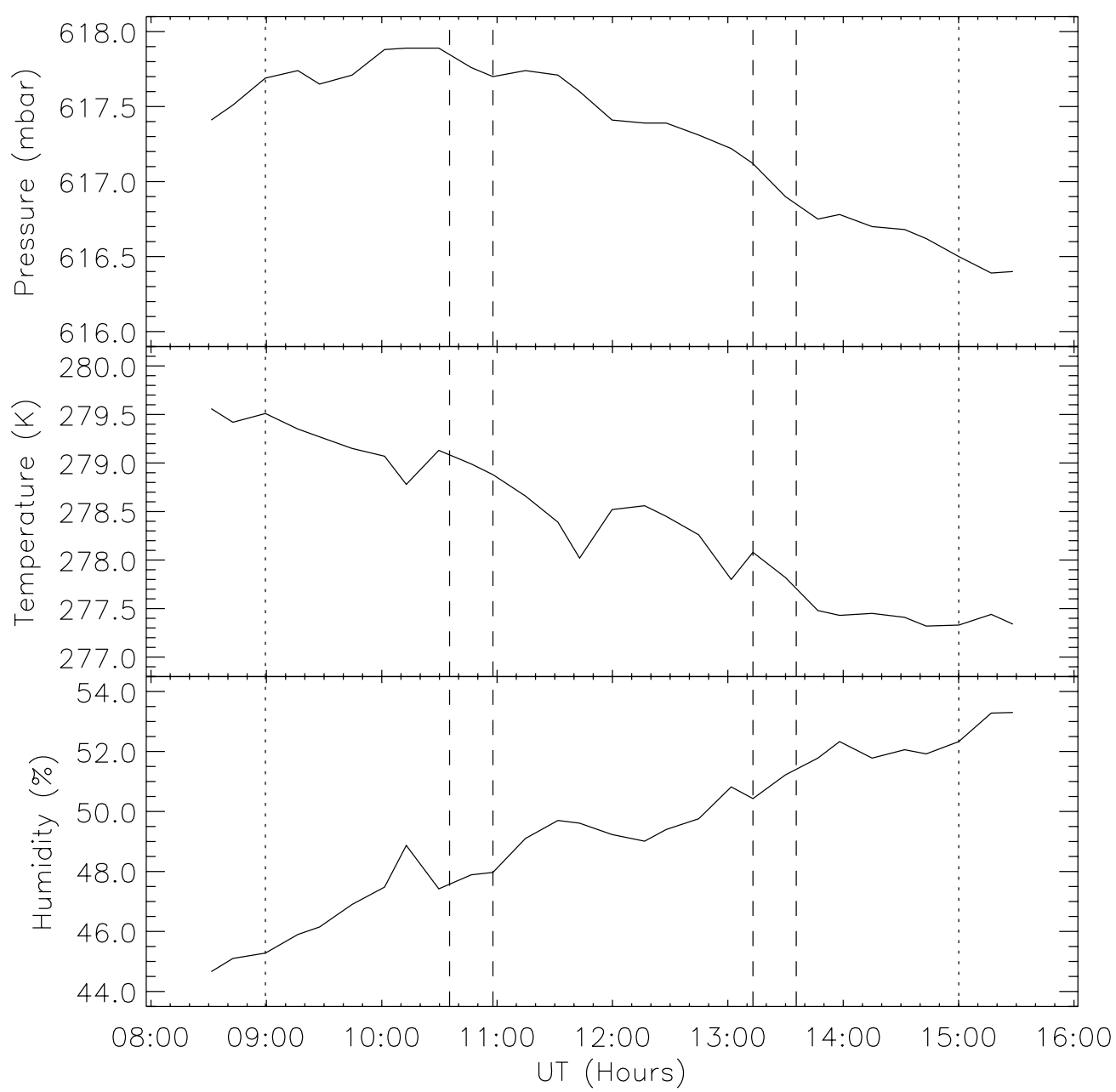

Figure 2. Air temperature (top), barometric air pressure (middle), and relative humidity (bottom) from UKIRT on Mauna Kea during stellar observations. The time axis is in Universal Time. The dotted lines show the approximate boundaries between HR 7906 and HD 209458 observations (09:00), and HD 209458 and HR 8634 observations (15:00). The dashed lines show the approximate times of the HD 209458 transit first, second, third, and fourth contacts.

ending at $100 \mathrm{~km}$, at various zenith angles corresponding to the air masses of particular science observations; aerosol extinction was calculated using the software-supplied Navy Maritime model; a user-supplied atmosphere based on the softwaresupplied tropical model $\left(15^{\circ}\right.$ north) was used. More details about the user-supplied atmosphere immediately follow.

Initial PcLnWin trials with the software-supplied tropical model atmosphere produced water lines that were about a factor of 3 too weak compared with real water features observed in the spectra of HD 209458, HR 7906, and HR 8634. The $4.2 \mathrm{~km}$ elevation of the Keck Observatory rises above most of the low-altitude water. The tropical model, the only softwaresupplied model to show a high-altitude (about 14-19 km) layer of increased humidity (Table 1), was modified to include increased humidity immediately above the altitude of Keck. Two additional layer boundaries were added at 4.2 and $4.5 \mathrm{~km}$. The temperature and pressure at the $4.2 \mathrm{~km}$ boundary were set to the approximate average of the UKIRT-supplied weather data. The temperature and pressure in the $4.5 \mathrm{~km}$ boundary were linearly interpolated between the values of the $4.2 \mathrm{~km}$ boundary and $5 \mathrm{~km}$ boundary. The humidity from 4.5 to $9 \mathrm{~km}$ was modified by adding a high-humidity peak at $5 \mathrm{~km}$, and allowing the humidity to fall off as an asymmetric Gaussian in the region between 4.5 and $9 \mathrm{~km}$.
For each science spectrum, we found the model of the terrestrial atmosphere that produces a theoretical spectrum which best fits the observed water lines. Using the tropical model as a template, many atmospheric models that increase the relative humidity in the $5 \mathrm{~km}$ altitude layer were produced. The models were first tested on a rapidly rotating B star spectrum at the highest zenith angle (HR 7906 at 40.5). A B star's rapid rotation smears out its own stellar lines via Doppler broadening, enabling the narrow terrestrial atmospheric lines to stand out in high contrast. The amount of humidity necessary to fit the data's water lines was not known a priori, so a batch of models was produced where the humidity in the $5 \mathrm{~km}$ altitude layer varies from $55 \%$ to $95 \%$. To match the resolutions of the models and the data, the model spectra were convolved with a Gaussian that mimics a resolving power of 68,400 . The best-fit humidity range was $65 \%-75 \%$.

Next, the HR 8634 and HD 209458 spectra were fit with models. Each science exposure was first continuum-normalized with the mean flat-field lamp spectrum, then with an eighth degree polynomial, and the models were continuum-normalized with a first-degree polynomial. (The PcLnWin models include the effects of Rayleigh scattering, which more strongly scatters blue wavelengths out of the transmitted light. This effect is only a few \% across the $87 \AA$ HIRES CCD width, so a first degree 
Table 1

PcLnWin Tropical Model

\begin{tabular}{|c|c|c|c|c|c|}
\hline $\begin{array}{l}\text { Boundary } \\
\text { No. }\end{array}$ & $\begin{array}{c}\text { Altitude } \\
(\mathrm{km})\end{array}$ & $\begin{array}{c}\text { Temperature } \\
\text { (C) }\end{array}$ & $\begin{array}{c}\text { Pressure } \\
\text { (mbar) }\end{array}$ & $\begin{array}{c}\text { Humidity } \\
\text { (Original) } \\
\text { (\% rel.) }\end{array}$ & $\begin{array}{c}\text { Humidity } \\
\text { (Modified) } \\
(\% \text { rel.) }\end{array}$ \\
\hline 1 & 0.0 & 300 & 1010.00 & 75.50 & 75.50 \\
\hline 2 & 1.0 & 294 & 904.00 & 72.80 & 72.80 \\
\hline 3 & 2.0 & 288 & 805.00 & 74.50 & 74.50 \\
\hline 4 & 3.0 & 284 & 715.00 & 48.30 & 48.30 \\
\hline 5 & 4.0 & 277 & 633.00 & 34.90 & 34.90 \\
\hline 6 & $(4.2)$ & (279) & $(617.00)$ & $\ldots$ & $(50.00)$ \\
\hline 7 & (4.5) & (275) & $(600.00)$ & $\ldots$ & (64.10) \\
\hline 8 & 5.0 & 270 & 559.00 & 37.70 & 70.00 \\
\hline 9 & 6.0 & 264 & 492.00 & 34.80 & 66.80 \\
\hline 10 & 7.0 & 257 & 432.00 & 32.00 & 58.10 \\
\hline 11 & 8.0 & 250 & 378.00 & 29.50 & 46.00 \\
\hline 12 & 9.0 & 244 & 329.00 & 25.40 & 33.20 \\
\hline 13 & 10.0 & 237 & 286.00 & 19.50 & 19.50 \\
\hline 14 & 11.0 & 230 & 247.00 & 13.20 & 13.20 \\
\hline 15 & 12.0 & 224 & 213.00 & 9.25 & 9.25 \\
\hline 16 & 13.0 & 217 & 182.00 & 5.88 & 5.88 \\
\hline 17 & 14.0 & 210 & 156.00 & 7.41 & 7.41 \\
\hline 18 & 15.0 & 204 & 132.00 & 9.92 & 9.92 \\
\hline 19 & 16.0 & 197 & 111.00 & 16.80 & 16.80 \\
\hline 20 & 17.0 & 195 & 93.70 & 19.20 & 19.20 \\
\hline 21 & 18.0 & 199 & 78.90 & 8.32 & 8.32 \\
\hline 22 & 19.0 & 203 & 66.60 & 3.75 & 3.75 \\
\hline 23 & 20.0 & 207 & 56.50 & 1.82 & 1.82 \\
\hline 24 & 21.0 & 211 & 48.00 & 0.92 & 0.92 \\
\hline 25 & 22.0 & 215 & 40.90 & 0.50 & 0.50 \\
\hline 26 & 23.0 & 217 & 35.00 & 0.33 & 0.33 \\
\hline 27 & 24.0 & 219 & 30.00 & 0.24 & 0.24 \\
\hline 28 & 25.0 & 221 & 25.70 & 0.16 & 0.16 \\
\hline 29 & 30.0 & 232 & 12.20 & 0.03 & 0.03 \\
\hline 30 & 35.0 & 243 & 6.00 & 0.01 & 0.01 \\
\hline 31 & 40.0 & 254 & 3.05 & $1.35 \mathrm{E}-5$ & $1.35 \mathrm{E}-5$ \\
\hline 32 & 50.0 & 270 & $8.54 \mathrm{E}-1$ & $4.11 \mathrm{E}-6$ & $4.11 \mathrm{E}-6$ \\
\hline 33 & 70.0 & 219 & $5.80 \mathrm{E}-2$ & $2.58 \mathrm{E}-7$ & $2.58 \mathrm{E}-7$ \\
\hline 34 & 100.0 & 191 & $2.89 \mathrm{E}-4$ & $1.31 \mathrm{E}-10$ & $1.31 \mathrm{E}-10$ \\
\hline
\end{tabular}

Note. The model atmosphere values for the sixth and seventh boundaries are enclosed in parentheses to denote that those boundaries are not present in the original tropical model.

polynomial is sufficient to correct for it over this range, even though Rayleigh scattering goes as $\lambda^{-4}$.)

Then we determined a wavelength solution by identifying atmospheric lines that appear in both the science exposure and a model spectrum. Wavelengths were calibrated in vacuum wavenumber (also called the "frequency reciprocal" $k=\frac{1}{\lambda} \times$ $\left.\frac{1}{n_{\text {medium }}}\right)$. We used the pixel values from the science exposure and the wavenumbers from the model spectrum to fit a sixth degree function that gives the wavenumber as a function of pixel in the science exposure. All the spectra were first calibrated to the vacuum "rest frame" of the atmospheric water lines. The Earth's motion causes the stellar lines to shift in relative velocity by about a third of a pixel over the course of the night, but this complication was easily corrected. We created a new linear wavenumber array with 20 times more elements than the data array. Then the science exposure and the model spectrum were spline-interpolated onto the new linear wavenumbers, which conserves flux at a level of $1 \times 10^{-4}$ across the entire chip.

Finally, the science exposures were divided by the models. In HR 8634, if the atmospheric lines were completely removed, only the continuum remained with noise scattered around a mean value of 1 . Underfitting the atmospheric lines left absorption
Table 2

Observing Log and PcLnWin Model Fitting: B Stars

\begin{tabular}{lccccccc}
\hline \hline Object & $\begin{array}{c}\text { Midtime } \\
(\text { UT })\end{array}$ & AM & $\begin{array}{c}\text { Humidity } \\
(\%)\end{array}$ & Object & $\begin{array}{c}\text { Midtime } \\
(\text { UT })\end{array}$ & AM & $\begin{array}{c}\text { Humidity } \\
(\%)\end{array}$ \\
\hline HR 7906 & $08: 38: 00$ & 1.32 & 69 & HR 8634 & $15: 11: 35$ & 1.12 & 70 \\
HR 7906 & $08: 40: 58$ & 1.30 & 69 & HR 8634 & $15: 12: 36$ & 1.12 & 68 \\
HR 7906 & $08: 42: 07$ & 1.30 & 69 & HR 8634 & $15: 13: 37$ & 1.12 & 70 \\
HR 7906 & $08: 43: 16$ & 1.29 & 68 & HR 8634 & $15: 14: 38$ & 1.12 & 70 \\
HR 7906 & $08: 44: 25$ & 1.29 & 68 & HR 8634 & $15: 15: 36$ & 1.12 & 68 \\
HR 7906 & $08: 45: 34$ & 1.28 & 69 & HR 8634 & $15: 16: 38$ & 1.13 & 68 \\
HR 7906 & $08: 46: 30$ & 1.28 & 68 & HR 8634 & $15: 17: 37$ & 1.13 & 70 \\
HR 7906 & $08: 47: 39$ & 1.27 & 69 & HR 8634 & $15: 18: 38$ & 1.13 & 70 \\
HR 7906 & $08: 48: 48$ & 1.27 & 67 & HR 8634 & $15: 19: 37$ & 1.13 & 68 \\
HR 7906 & $08: 49: 57$ & 1.26 & 68 & HR 8634 & $15: 20: 38$ & 1.14 & 68 \\
HR 7906 & $08: 51: 06$ & 1.26 & 67 & HR 8634 & $15: 21: 38$ & 1.14 & 68 \\
HR 8634 & $15: 05: 24$ & 1.10 & 69 & HR 8634 & $15: 22: 38$ & 1.14 & 70 \\
HR 8634 & $15: 06: 34$ & 1.11 & 70 & HR 8634 & $15: 23: 38$ & 1.14 & 68 \\
HR 8634 & $15: 07: 35$ & 1.11 & 67 & HR 8634 & $15: 24: 40$ & 1.15 & 68 \\
HR 8634 & $15: 08: 34$ & 1.11 & 69 & HR 8634 & $15: 25: 40$ & 1.15 & 69 \\
HR 8634 & $15: 09: 34$ & 1.11 & 68 & HR 8634 & $15: 26: 40$ & 1.15 & 68 \\
HR 8634 & $15: 10: 35$ & 1.11 & 68 & $\ldots$ & $\ldots$ & $\ldots$ & $\ldots$ \\
\hline
\end{tabular}

lines that stand out from the continuum, and overfitting the atmospheric lines resulted in apparent emission lines that stood out from the continuum. We used

$$
\chi^{2}=\Sigma\left[\left(\operatorname{model}_{i}-\operatorname{data}_{i}\right)^{2} / \operatorname{data}_{i}\right]
$$

as a goodness-of-fit parameter. Typical values for $\chi^{2}$ were 0.4 0.6 for the HR 8634 spectra and for the red half of the HD 209458 spectra (which does not contain the deep $\mathrm{NaD}$ doublet). Note that this formulation of $\chi^{2}$ is not the same as the reduced $\chi^{2}$, which has a best-fit value of unity. In continuum regions with no stellar absorption lines, the best-fit value for our definition of $\chi^{2}$ in the no-noise limit would be zero. Nonzero contributions to $\chi^{2}$ come from noise, as well as stellar absorption lines that do not appear in the telluric model. These values for $\chi^{2}$ correspond to a noise level of $(1.0-1.5) \times 10^{-5}$ compared to the normalized continuum value. The science spectra have approximately (12) $\times 10^{-5}$ counts pixel $^{-1}$, which corresponds to a photon-noise limit of $(0.5-1.0) \times 10^{-5}$. Thus, we are nearly, but not quite, photon-noise limited. The best-fit humidity for each science exposure is recorded in Tables 2 and 3.

Upon closer examination, after removing atmospheric water lines using the HITRAN models described above, HR 7906 shows interstellar absorption in the $\mathrm{NaD}$ doublet, with peak absorption offset approximately $1 \AA$ from the $\mathrm{NaD}$ doublet in HD 209458. In fact, HR 7906 (also known as $\alpha$ Del) was used (Welsh et al. 1991, 1998) to map the boundaries of the Local Bubble. When placed on the Welsh et al. (1998) plots, which use updated stellar distances from Hipparcos, HR 8634 sits just inside the sodium boundary of the Local Bubble, explaining why it does not show interstellar $\mathrm{Na}$ in our spectra. Thus, HR 7906 was discarded and only HR 8634 was used as a reference star in two different ways for the analysis described further in Section 3. The first option was to scale the line strengths linearly with air mass to match the air masses of the science exposures. The second option was to scale the line depths from the reference B star spectrum by a range of values, and use the value that produces minimal residuals, which would allow the line strengths to grow approximately exponentially with increasing air mass.

Next, all the spectra of HD 209458 were shifted onto the same velocity frame as the first HD 209458 exposure by 
Table 3

Observing Log and PcLnWin Model Fitting: HD 209458

\begin{tabular}{|c|c|c|c|c|c|c|c|c|c|}
\hline $\begin{array}{l}\text { Midtime } \\
\text { (UT) }\end{array}$ & $\mathrm{AM}$ & $\begin{array}{l}\text { TFTC } \\
\text { (days) } \\
\end{array}$ & Transit & $\begin{array}{c}\text { Humidity } \\
(\%)\end{array}$ & $\begin{array}{c}\text { Midtime } \\
\text { (UT) }\end{array}$ & $\mathrm{AM}$ & $\begin{array}{l}\text { TFTC } \\
\text { (days) }\end{array}$ & Transit & $\begin{array}{c}\text { Humidity } \\
(\%)\end{array}$ \\
\hline 09:19:40 & 1.56 & -0.11363 & Out & 77 & $12: 21: 13$ & 1.01 & 0.00909 & In & 71 \\
\hline 09:25:00 & 1.52 & -0.10988 & Out & 70 & $12: 26: 03$ & 1.01 & 0.01246 & In & 70 \\
\hline $09: 29: 52$ & 1.48 & -0.10651 & Out & 69 & $12: 30: 54$ & 1.01 & 0.01585 & In & 71 \\
\hline 09:34:44 & 1.45 & -0.10312 & Out & 71 & $12: 35: 44$ & 1.00 & 0.01922 & In & 72 \\
\hline $09: 39: 35$ & 1.42 & -0.09976 & Out & 71 & $12: 40: 34$ & 1.00 & 0.02259 & In & 70 \\
\hline $09: 44: 23$ & 1.40 & -0.09639 & Out & 73 & $12: 45: 25$ & 1.00 & 0.02596 & In & 70 \\
\hline $09: 49: 14$ & 1.37 & -0.09301 & Out & 72 & $12: 57: 22$ & 1.00 & 0.02933 & In & 71 \\
\hline 09:57:01 & 1.33 & -0.08763 & Out & 73 & $13: 02: 15$ & 1.00 & 0.04094 & In & 72 \\
\hline $10: 01: 55$ & 1.31 & -0.08426 & Out & 72 & 13:07:01 & 1.00 & 0.04431 & In & 71 \\
\hline $10: 06: 46$ & 1.29 & -0.08089 & Out & 73 & $13: 11: 55$ & 1.00 & 0.04770 & Egress & 73 \\
\hline $10: 11: 35$ & 1.27 & -0.07751 & Out & 73 & $13: 16: 46$ & 1.01 & 0.05106 & Egress & 73 \\
\hline $10: 16: 27$ & 1.25 & -0.07414 & Out & 74 & $13: 21: 38$ & 1.01 & 0.05444 & Egress & 70 \\
\hline $10: 21: 16$ & 1.23 & -0.07076 & Out & 73 & $13: 26: 30$ & 1.01 & 0.05781 & Egress & 70 \\
\hline $10: 26: 15$ & 1.21 & -0.06738 & Out & 72 & $13: 31: 20$ & 1.01 & 0.06118 & Egress & 72 \\
\hline $10: 31: 05$ & 1.20 & -0.06402 & Out & 70 & $13: 36: 11$ & 1.02 & 0.06455 & Out & 71 \\
\hline $10: 35: 54$ & 1.18 & -0.06064 & Ingress & 73 & $13: 41: 03$ & 1.02 & 0.06793 & Out & 76 \\
\hline $10: 40: 46$ & 1.17 & -0.05728 & Ingress & 73 & $13: 45: 55$ & 1.02 & 0.07131 & Out & 72 \\
\hline $10: 45: 38$ & 1.15 & -0.05391 & Ingress & 73 & $13: 50: 46$ & 1.03 & 0.07468 & Out & 72 \\
\hline $10: 50: 28$ & 1.14 & -0.05054 & Ingress & 70 & $13: 55: 37$ & 1.04 & 0.07806 & Out & 71 \\
\hline $10: 55: 17$ & 1.13 & -0.04717 & Ingress & 72 & $14: 00: 30$ & 1.04 & 0.08143 & Out & 73 \\
\hline 11:00:13 & 1.12 & -0.04380 & In & 71 & $14: 05: 22$ & 1.05 & 0.08482 & Out & 71 \\
\hline $11: 05: 02$ & 1.11 & -0.04042 & In & 72 & $14: 10: 14$ & 1.05 & 0.08818 & Out & 72 \\
\hline 11:09:57 & 1.10 & -0.03705 & In & 71 & $14: 15: 01$ & 1.06 & 0.09155 & Out & 71 \\
\hline $11: 14: 47$ & 1.09 & -0.03368 & In & 75 & $14: 19: 54$ & 1.07 & 0.09493 & Out & 69 \\
\hline $11: 42: 17$ & 1.05 & -0.01790 & In & 71 & $14: 24: 45$ & 1.08 & 0.09832 & Out & 69 \\
\hline 11:47:09 & 1.04 & -0.01454 & In & 70 & $14: 29: 41$ & 1.09 & 0.10167 & Out & 69 \\
\hline $11: 52: 00$ & 1.04 & -0.01117 & In & 70 & $14: 34: 30$ & 1.10 & 0.10506 & Out & 73 \\
\hline $11: 56: 54$ & 1.03 & -0.00779 & In & 71 & $14: 39: 20$ & 1.11 & 0.10843 & Out & 69 \\
\hline $12: 01: 44$ & 1.03 & -0.00441 & In & 70 & $14: 44: 13$ & 1.12 & 0.11180 & Out & 70 \\
\hline $12: 06: 36$ & 1.02 & -0.00104 & In & 72 & $14: 49: 04$ & 1.13 & 0.11517 & Out & 72 \\
\hline $12: 11: 30$ & 1.02 & 0.00233 & In & 72 & $14: 53: 54$ & 1.14 & 0.11853 & Out & 70 \\
\hline $12: 16: 19$ & 1.01 & 0.00571 & In & 71 & $14: 58: 46$ & 1.15 & 0.12191 & Out & 71 \\
\hline
\end{tabular}

cross-correlating the remaining stellar lines and shifting. Then the vacuum wavenumbers were converted into the observed wavelength to aid in identifying absorption lines, assuming an air index of refraction of 1.00017538 .

\subsubsection{Cosmic Rays}

During the initial data reduction, cosmic rays were removed by fitting a low-order polynomial to each individual pixel's behavior in time, and replacing corrupted pixels with the value of the polynomial that was fitted. But the unpredictability of the continuum blaze function over time meant that the time series for an individual pixel could not be fit with a smooth low-order polynomial. Therefore, some cosmic rays remained. Another iteration of removing cosmic rays was made after spectra were continuum-normalized and the atmosphere was removed. To test for the presence of cosmic rays, each pixel's value is compared with the mean of that pixel's values in the exposure prior and the exposure afterward. For the first and last exposures, the pixels are compared with their value in the second and next-tolast exposures, respectively. Any pixels which deviate above a certain threshold from the mean of their neighbor exposures' pixels are replaced with the value of the mean of their neighbor exposures' pixels.

\subsubsection{Monte Carlo Uncertainty Estimation}

Given all of the uncertainties associated with the additional reduction steps described above, one needs to know how those uncertainties affect the reliability of the final results. To explore the realm of uncertainty, equal-probability Monte Carlo calculations were performed during the final data reduction. There are four different reduction parameters that have a range of possible values, and random values for each of the parameters were selected. When randomly selecting the value for a parameter, each possible value is given equal weight. 50 iterations of the Monte Carlo parameter selection process were performed. The four parameters and their possible values are as follows.

1. Continuum identification: due to the unfortunate necessity that a continuum mask be constructed by hand, subjective error is introduced. The Monte Carlo code picks a random integer between -2 and 2 , which is the number of pixels that the baseline continuum mask is shifted by before fitting a polynomial to the masked region.

2. Continuum blaze normalizing: the Monte Carlo code randomly picks between the integers 7 and 9 . The integer is then used to fit the continuum identified in the previous step by an seventh, eighth, or ninth degree polynomial.

3. Terrestrial atmospheric line removal: to review, there are three different methods that are used for removing atmospheric lines: scaling the atmospheric lines in HR 8634 linearly with air mass, scaling the atmospheric lines in HR 8634 by arbitrary factors and choosing the factor that produces the minimum residuals, and fitting the atmospheric lines with theoretical model atmospheres. The Monte Carlo 
code picks a random integer from 1 to 3 , which controls which method to employ.

4. Cosmic ray removal: after continuum normalizing, the Monte Carlo code picks a random number between 1.001 and 1.010, which is the cosmic ray removal threshold. For each exposure, any pixels which have values greater than the threshold compared with the exposure immediately before and immediately after are set to the mean of the pixel's value in the exposure before and the exposure after.

\section{ANALYSIS}

\subsection{Method}

By continuum-normalizing all of the spectra, where the continuum in each of the spectra has been normalized to 1 , all spectra are also conveniently normalized to remove global flux losses from the transiting planet's eclipse of the star. The light curve of the HD 209458b transit is well known (Brown et al. 2001), and can reach a depth of $1.6 \%$ relative to the stellar flux out of transit. It is counterproductive and unnecessary to renormalize the spectra to match the well-known transit light curve. With all of the spectra normalized to a continuum value of 1 , the $\mathrm{NaD}$ flux from the star obscured by the disk of the planet is multiplied back in "for free," so the effect of the planet's disk is undetectable. Any remaining detectable absorption difference in a molecular or atomic line is exclusively due to transmission through the planet's atmosphere.

There may be additional limb-darkening effects that need to be understood. Unfortunately, the necessary removal of echelle blaze effects by continuum-normalizing the spectra renders the measurement of any possible wavelength-dependent limbdarkening impossible.

\subsection{Bandwidths}

Because C2002 did not know a priori the width of NaD absorption that could be found, they examined three different spectral bands centered on the $\mathrm{NaD}$ lines. To facilitate comparison with C2002, this work uses a similar method, with one difference. Spectral bands progressively get narrower to concentrate on the $\mathrm{NaD}$ lines, but a linear estimate of the limb-darkening is not included because any linear component of limb darkening was removed in the continuum-normalizing step. In this work, the C2002-medium and C2002-narrow bands were reproduced. It was not possible to reproduce the $\mathrm{C} 2002$-wide band because its short-wavelength end was off the CCD chip in this particular HIRES configuration. The short-wavelength limit of the medium band falls just inside the edge of the CCD. HIRES allows a higher resolution view of the $\mathrm{NaD}$ lines, so this work is also able to reproduce the narrower bands used by N2005 and S2008 (Table 4).

\subsection{Results}

For each Monte Carlo iteration $\left(N_{\text {total }}=50\right)$, a partial result for the relative flux in and out of transit, and a partial error are calculated as follows:

$$
\begin{gathered}
\text { Partial Result }_{n}=\frac{\overline{\text { fluxes }_{\text {in } n}}-\overline{\text { fluxes }_{\text {out } n}}}{\overline{\text { fluxes }_{\text {out } n}}}, \\
\text { Partial Error }_{n}=\sqrt{\left(\frac{\sigma_{\text {in }}}{\overline{\text { fluxes }_{\text {out } n}}}\right)^{2}+\left(\frac{\sigma_{\text {out }} \overline{\text { fluxes }_{\text {in } n}}}{\overline{\text { fluxes }_{\text {out }} n}}\right)^{2}},
\end{gathered}
$$

Table 4

Definition of Wavelength Bands

\begin{tabular}{lccl}
\hline \hline Name & $\begin{array}{c}\text { Range } \\
(\AA)\end{array}$ & $\begin{array}{c}\text { Range } \\
\left(\mathrm{cm}^{-1}\right)\end{array}$ & Compare Results With \\
\hline Medium & $5874.00-5912.00$ & $16912.44-17020.56$ & $\mathrm{C} 2002$ \\
Narrow & $5887.00-5899.00$ & $16932.36-16983.57$ & $\mathrm{C} 2002$ \\
$2.0 \AA$ & $5895.06-5897.06$ & $16955.20-16960.90$ & $\mathrm{~N} 2005$ \\
$2.0 \AA$ & $5889.09-5891.09$ & $16972.40-16978.10$ & $\mathrm{~N} 2005$ \\
$0.3 \AA$ & $5895.91-5896.21$ & $16957.62-16958.48$ & $\mathrm{~N} 2005$ \\
$0.3 \AA$ & $5889.94-5890.24$ & $16974.81-16975.67$ & $\mathrm{~N} 2005$ \\
\hline
\end{tabular}

Note. The Wide band from C2002 cannot be used in this work because it falls off the edge of the HIRES detector.

where the index $n$ represents each Monte Carlo iteration of the secondary data reduction procedure and $\sigma$ 's are the standard deviations (s.d.s) of the means (over 21 in-transit spectra and 33 out-of-transit spectra). The total results in this work are calculated by:

$$
\text { Result }=\overline{(\text { Partial Results })_{n}} .
$$

The total errors in this work can be calculated by several different ways. The smallest possible error will usually be the s.d. of the mean of the results (SDOM). A large error will be the partial errors propagated through a straight quadrature sum. An intermediate-sized error will be the mean of all the partial errors.

Results from this work are shown in Table 5 (comparison with C2002), Table 6 (comparison with N2005), and Table 7 (comparison with S2008). The errors quoted in the tables are the means of the partial errors. Nonsodium lines were also examined at a $2.0 \AA$ wide band, and a $0.3 \AA$ wide band (the width that fits around each weak line most tightly while excluding as much continuum as possible), and the results are also shown in Table 6.

For nonsodium lines, $\mathrm{C} 2002$ detected no extra absorption that is more significant than $1.5 \sigma$. Note that where $\mathrm{C} 2002$ was just able to resolve these lines, HIRES has enough resolution to completely resolve the weak $\mathrm{Ni}$ I line in between the $\mathrm{C} 2002$ $\mathrm{NaD}$ bands and to also reveal that some weak lines at lower resolution are actually overlapping lines of two completely different species. In the C2002 work, the lower Space Telescope Imaging Spectrograph (STIS) resolution and the choice of the narrow band resulted in significant continuum saturation of any absorption in the line. This continuum saturation effect is identical to that which is seen in the C2002-medium band around the $\mathrm{NaD}$ doublet in this work. $\mathrm{C} 2002$ found no significant absorption in the wide band because an appreciable amount of continuum was mixed along with sodium into the flux averaging. If the bands for nonsodium lines are narrowed to exclude continuum, any weak absorption signals are more likely to be found.

There could be cause for concern if all lines in the reduced spectra show absorption when enough continuum is excluded. As shown in Table 6 however, the only nonsodium line whose results appear to get more significant when more continuum is excluded is Fe I $5884 \AA$. By contrast, the Fe I 5906, 5910, and $5914 \AA$ results get less significant as more continuum is excluded, probably due to lingering residuals in the continuumnormalizing process.

The dramatic and recent results of S2008 are measured for bands of $3 \AA, 1.5 \AA$, and $0.75 \AA$ centered on each of the $\mathrm{NaD}$ 
Table 5

$\mathrm{NaD}$ Results Compared with C2002

\begin{tabular}{lccc}
\hline \hline $\begin{array}{l}\text { Feature } \\
(\AA)\end{array}$ & Wide Band & $\begin{array}{c}\text { Medium Band } \\
\text { Significances }(\sigma)\end{array}$ & Narrow Band \\
\hline $\mathrm{NaD} 5889.97,5895.94$ & $(-3.1 \pm 3.6) \times 10^{-5}$ & $(-13.1 \pm 3.8) \times 10^{-5}$ & $(-23.2 \pm 5.7) \times 10^{-5}$ \\
$(\mathrm{C} 2002)$ & 0.9 & 3.4 & 4.1 \\
$\mathrm{NaD} 5889.97,5895.94$ & $\ldots$ & $(-37.8 \pm 7.4) \times 10^{-5}$ & $(-108.8 \pm 25.7) \times 10^{-5}$ \\
$($ This work $)$ & $\cdots$ & 5.09 & 4.23 \\
\hline
\end{tabular}

Table 6

$\mathrm{NaD}$ Results Compared with N2005

\begin{tabular}{|c|c|c|c|c|c|}
\hline $\begin{array}{l}\text { Feature } \\
(\AA)\end{array}$ & $4.0 \AA$ & $2.0 \AA$ & $\begin{array}{c}1.2 \AA \\
\text { Significances }(\sigma)\end{array}$ & $0.6 \AA$ & $0.3 \AA$ \\
\hline $\mathrm{NaD} 5890$ & $\ldots$ & $-2 \pm 6$ & $\ldots$ & $\cdots$ & $-70 \pm 28$ \\
\hline$(\mathrm{N} 2005)$ & $\ldots$ & 0.33 & $\ldots$ & $\ldots$ & 2.5 \\
\hline $\mathrm{NaD} 5890$ & $-6.9 \pm 4.0$ & $-13.9 \pm 3.9$ & $-7.6 \pm 4.1$ & $-2.4 \pm 7.4$ & $8.2 \pm 16.6$ \\
\hline (This work) & 1.73 & 3.60 & 1.85 & 0.33 & +0.49 \\
\hline $\mathrm{NaD} 5896$ & $\ldots$ & $6 \pm 9$ & $\ldots$ & $\ldots$ & $12 \pm 48$ \\
\hline$(\mathrm{N} 2005)$ & $\ldots$ & +0.67 & $\ldots$ & $\ldots$ & +0.25 \\
\hline NaD 5896 & $-20.5 \pm 4.1$ & $-25.4 \pm 5.4$ & $-27.4 \pm 6.7$ & $-28.7 \pm 8.6$ & $-17.7 \pm 14.6$ \\
\hline (This work) & 4.96 & 4.66 & 4.07 & 3.34 & 1.21 \\
\hline Other lines & & & & & \\
\hline Ni I 5893 & $\begin{array}{c}-13.2 \pm 4.1 \\
3.22\end{array}$ & $\begin{array}{c}-8.9 \pm 3.3 \\
2.86\end{array}$ & $\begin{array}{c}-9.4 \pm 3.7 \\
2.56\end{array}$ & $\begin{array}{c}-10.9 \pm 4.5 \\
2.39\end{array}$ & $\begin{array}{c}-20.7 \pm 7.1 \\
2.94\end{array}$ \\
\hline Fe I 5884 & $\begin{array}{c}-1.3 \pm 1.3 \\
0.99\end{array}$ & $\begin{array}{c}-0.5 \pm 1.6 \\
0.31\end{array}$ & $\begin{array}{c}-3.5 \pm 2.7 \\
1.32\end{array}$ & $\begin{array}{c}-3.9 \pm 2.4 \\
1.63\end{array}$ & $\begin{array}{c}-7.1 \pm 3.2 \\
2.21\end{array}$ \\
\hline Fe I 5906 & $\begin{array}{c}-4.8 \pm 1.7 \\
2.89\end{array}$ & $\begin{array}{c}-5.2 \pm 2.2 \\
2.29\end{array}$ & $\begin{array}{c}-4.5 \pm 2.4 \\
1.87\end{array}$ & $\begin{array}{c}-4.2 \pm 3.2 \\
1.33\end{array}$ & $\begin{array}{c}-4.0 \pm 4.1 \\
0.97\end{array}$ \\
\hline Fe I 5910 & $\begin{array}{l}1.4 \pm 1.1 \\
+1.27\end{array}$ & $\begin{array}{c}1.9 \pm 1.6 \\
+1.18\end{array}$ & $\begin{array}{l}2.3 \pm 2.4 \\
+1.02\end{array}$ & $\begin{array}{c}3.5 \pm 3.5 \\
+1.02\end{array}$ & $\begin{array}{c}-0.8 \pm 3.7 \\
0.22\end{array}$ \\
\hline Fe I $5914.1,5914.2$ & $\begin{array}{c}-5.7 \pm 2.0 \\
2.94\end{array}$ & $\begin{array}{c}-5.0 \pm 1.9 \\
2.62\end{array}$ & $\begin{array}{c}-8.6 \pm 2.8 \\
3.05\end{array}$ & $\begin{array}{c}-7.3 \pm 4.3 \\
1.69\end{array}$ & $\begin{array}{c}-6.8 \pm 7.3 \\
0.92\end{array}$ \\
\hline
\end{tabular}

lines, then both lines are combined. When the $\mathrm{S} 2008$ bands are applied to this Keck/HIRES data, our results are a factor of 2.7-3.0 larger in the $2 \times 3 \AA$ and $2 \times 1.5 \AA$ bands. There is agreement with the results of S2008 for the $2 \times 0.75 \AA$ band. In general, the results of S2008 imply that the sodium feature is narrower than the feature reported by our work. Our results for each line of the $\mathrm{NaD}$ doublet cannot be compared because S2008 did not supply individual differential fluxes.

To compare the results of this work with the results from previous work, careful attention must be paid to how the total errors were calculated. Both C2002 and N2002 calculated their results as differences of means of the relative fluxes.

$$
\text { Result }=\overline{\text { Relative fluxes }_{\text {in }}}-\overline{\text { Relative fluxes }_{\text {out }}} .
$$

In $\mathrm{C} 2002$, the relative fluxes were the difference between the integrated flux in the band of interest and the average of the integrated flux from adjacent bands. C2002's partial errors were the s.d. of the mean $\left(\sigma_{\text {in }}=\operatorname{SDOM}\left(\right.\right.$ relative fluxes $\left.s_{\text {in }}\right)$ and $\sigma_{\text {out }}=\operatorname{SDOM}\left(\right.$ relative fluxes $\left.\left._{\text {out }}\right)\right)$. In N2005, the relative fluxes were (flux-template)/template, where the template spectrum was an average of all the in-transit and out-of-transit spectra. N2005's partial errors were the s.d. C2002 used 171 in-transit and 207 out-of-transit spectra, while N2005 used 12 in-transit and 12 out-of-transit spectra, almost 16 times fewer spectra and a factor of 4 larger s.d.s of the mean. This work uses 21 in-transit and 33 out-of-transit spectra, an intermediate case.

Furthermore, C2002 and N2005 propagated their errors differently. C2002's total errors appear to be straight quadrature summed:

$$
\text { Total error }=\sqrt{\sigma_{\text {in }}^{2}+\sigma_{\text {out }}^{2}}=\sqrt{\frac{\text { s.d. }{ }_{\text {in }}^{2}}{N_{\text {in }}}+\frac{\text { s.d. }_{\text {out }}^{2}}{N_{\text {out }}}},
$$

which agrees with their quoted errors. Straight quadrature sum error propagation assumes that all the partial errors are uncorrelated. N2005 write the equation for their total error as

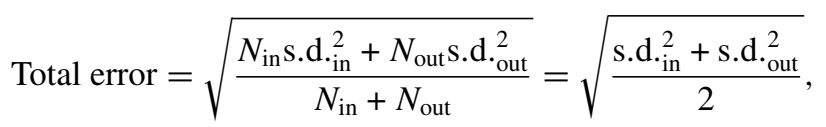

where $N_{\text {in }}=N_{\text {out }}=12$. Thus, $\mathrm{N} 2005$ will have a factor of 1.4 times smaller total errors, assuming that the identical partial errors are fed into the error propagation.

The variations in error calculations among the different researchers can be unified by using SDOM for the partial errors and straight quadrature sum error propagation (C2002 will not change, N2005 will). The s.d. represents uncertainty in the value of each measurement, and the s.d. of the mean represents the uncertainty in the mean of a collection of measurements, which converges as $\sqrt{N}$. N2005's total errors will increase by $\sqrt{2}$ and decrease by $\sqrt{12}$, for an overall decrease by a factor of $\sqrt{6}=2.45$. Then N2005's $2.5 \sigma$ absorption in a $0.3 \AA$ band of the $\mathrm{NaD} 5890$ line becomes a more compelling $6.1 \sigma$, but the new $0.6-1.6 \sigma$ results in N2005's other bands, which are still consistent with no detection, desire explanation. 
Table 7

NaD Results Compared with S2008

\begin{tabular}{lccc}
\hline \hline $\begin{array}{l}\text { Feature } \\
(\AA)\end{array}$ & $2 \times 3 \AA$ & $\begin{array}{c}2 \times 1.5 \AA \\
\text { Significances }(\sigma)\end{array}$ & $2 \times 0.75 \AA$ \\
\hline $\mathrm{NaD} 5889.97,5895.94$ & $(-5.6 \pm 0.7) \times 10^{-4}$ & $(-7.0 \pm 1.1) \times 10^{-4}$ & $(-13.5 \pm 1.7) \times 10^{-4}$ \\
$(\mathrm{~S} 2008)$ & 8.0 & 6.4 & 7.9 \\
$\mathrm{NaD}$ 5889.97, 5895.94 & $(-17 \pm 5) \times 10^{-4}$ & $(-19 \pm 5) \times 10^{-4}$ & $(-14 \pm 7) \times 10^{-4}$ \\
(This work) & 3.4 & 3.8 & 2.0 \\
\hline
\end{tabular}

\section{DISCUSSION}

As shown in Tables 2 and 3, the humidity which best fit the convolved HITRAN models to the data changes over the course of the night. Though humidity levels from the weather data from the night of the observation (see Figure 2) rose from about $48 \%$ to about $54 \%$, the best-fit humidity shows no such rise. This is possibly due to two causes: the model humidity in the $4.2 \mathrm{~km}$ layer being set at a constant $50 \%$, and a constant temperature was used in the models because the measured temperature variation throughout the night was less than $3 \mathrm{~K}$. HITRAN is capable of modeling different humidity and temperature profiles, to see if the best-fit models can be in better agreement with archive weather data from the night of observations.

When analyzing the results of the Monte Carlo data reduction procedure, it is desirable to understand the effects of the different methods used. Figures 3 and 4 display these Monte Carlo results for the medium and narrow bands used by C2002, respectively. For each band, Monte Carlo realizations with different continuum-normalizing polynomials and different atmosphere-removal techniques are plotted. Overall, the results and uncertainties are regularly distributed. The deeper the absorption, the larger the uncertainty, so the overall significance is $\approx 5 \sigma$ for the medium band and $\approx 4 \sigma$ for the narrow band for all possible reduction iterations. One concern in this work is the effect that continuum-normalizing has on the final reduced spectra. The figures show that in general, seventh degree polynomials produce stronger absorption, and eight and ninth degree polynomials produce progressively weaker absorption. Another concern is the removal of atmospheric water lines. The figures show that properly scaling the rapidly rotating B star HR 8634 by air mass and using the HITRAN models produce almost equal results. The figures also show spurious results with larger uncertainty from the arbitrary scaling method with HR 8634. Thus, arbitrary scaling of a rapidly rotating B star can be discarded as a method for removing the terrestrial atmosphere absorption lines.

The results in Table 5, $(-37.8 \pm 7.4) \times 10^{-5}$ in the medium band and $(-108.8 \pm 25.7) \times 10^{-5}$ in the narrow band, appear to confirm the presence of $\mathrm{Na}$ reported by $\mathrm{C} 2002$. However, looking at the magnitude of our detection and looking at each line individually introduces caution. The 4.23 and $5.09 \sigma$ detections of $\mathrm{C} 2002$ are only reported by us at $0.33-3.60 \sigma$ levels for the $5890 \AA$ line, and $1.21-4.96 \sigma$ for the $5896 \AA$ line. Additionally, that the $0.3 \AA$ band results are weaker than the 2.0 $\AA$ band results is worrisome, if the planetary absorption feature is narrow. However, the planet's radial velocity varies over the $2 \mathrm{hr}$ of the transit from about -10 to $+10 \mathrm{~km} \mathrm{~s}^{-1}$, which would make the planetary absorption feature move from -0.2 to +0.2 $\AA$. At the extreme ends of the transit, the deepest parts of the absorption features are simply Doppler shifted out of the $0.3 \AA$ bandpass. If the method used in this work is robust, one could measure the effective width of the planetary absorption by both

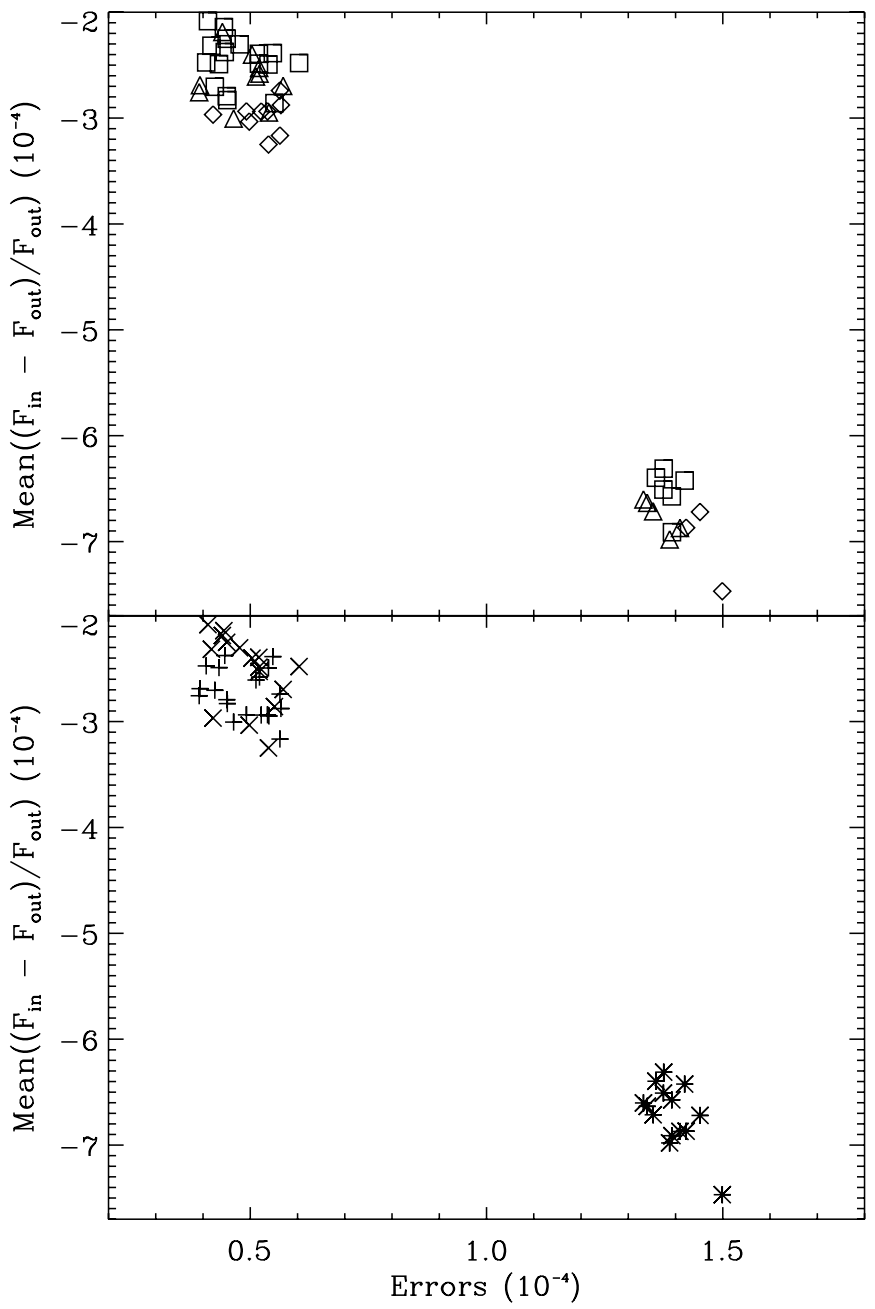

Figure 3. Monte Carlo results and uncertainties for the C2002-medium band. On the top, different continuum-normalizing polynomials—diamonds: seventh degree, triangles: eighth, squares: ninth. On the bottom, different atmosphere removal processes - plusses: air-mass scaling HR 8634, stars: arbitrary scaling HR 8634, crosses: HITRAN models. The results quoted in Table 5 are the means of each of these distributions. The results and the uncertainties appear to be regularly distributed.

predicting the absorption's wavelength from the planet's radial velocity and decreasing the tested bandwidth until the measured $\mathrm{S} / \mathrm{N}$ peaks, in between continuum contamination at large scales and instrument noise at small scales. Table 5 estimates a $2 \AA$ effective width for the $5890 \AA$ line and a $4 \AA$ effective width for the $5896 \AA$ Aline.

The robustness of the reduction and analysis procedure can be tested by measuring non-Na lines. None of the other lines tested in C2002 showed any appreciable additional absorption using a medium $(38 \AA$ ) or narrow $(12 \AA)$ bandpass. Note that high-resolution spectra reveal that these kinds of other lines are 


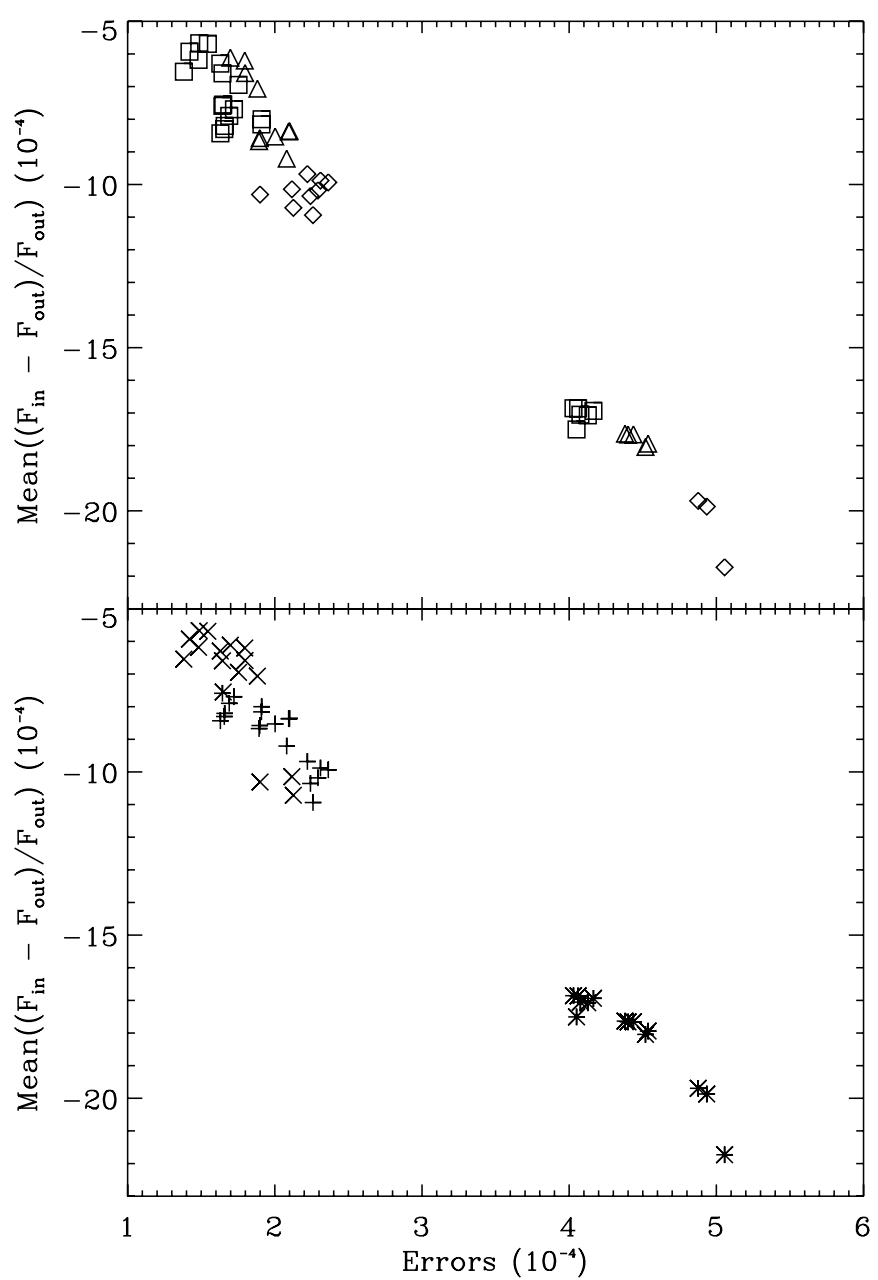

Figure 4. Monte Carlo results and uncertainties for the $\mathrm{C} 2002$ narrow band On the top, different continuum-normalizing polynomials-diamonds: seventh degree, triangles: eighth, squares: ninth. On the bottom, different atmosphere removal processes-plusses: air-mass scaling HR 8634, stars: arbitrary scaling HR 8634, crosses: HITRAN models. The results quoted in Table 5 are the means of each of these distributions. The results and the uncertainties appear to be regularly distributed.

intrinsically quite narrow (less than $1 \AA$ ), so the C2002 "offsource" measurements may be contaminated with much noncontributing continuum. The total results and uncertainties for other lines tested in this work are shown in Table 6. In Figure 7, two lines that show no additional absorption ( $\mathrm{Fe}$ I 5884 and Fe I $5910 \AA$ ) have Monte Carlo result distributions that are random, have results that show both absorption (negative) and emission (positive), and have mean results near zero.

Based on the total results alone, three of the lines appear to also show some absorption: Ni I 5893, Fe I 5906, and Fe I 5914 A. The distributions of the Monte Carlo runs for these lines are shown in Figure 6. For Ni I 5893 and Fe I $5914 \AA$, the strongest absorption signatures result from arbitrarily scaling HR 8634 (denoted by the stars in the figure). Again, the arbitrary scaling of HR 8634 produces results that disagree with the two other sky-subtraction methods. When these points are excluded from the analysis, the apparent absorption signatures in these lines weaken substantially.

Another way to estimate the effects of systematic errors is to randomly choose subsets of the in-transit and out-of-transit spectra and to repeat the analysis. However, that approach is better suited for data sets with large numbers of spectra. It is

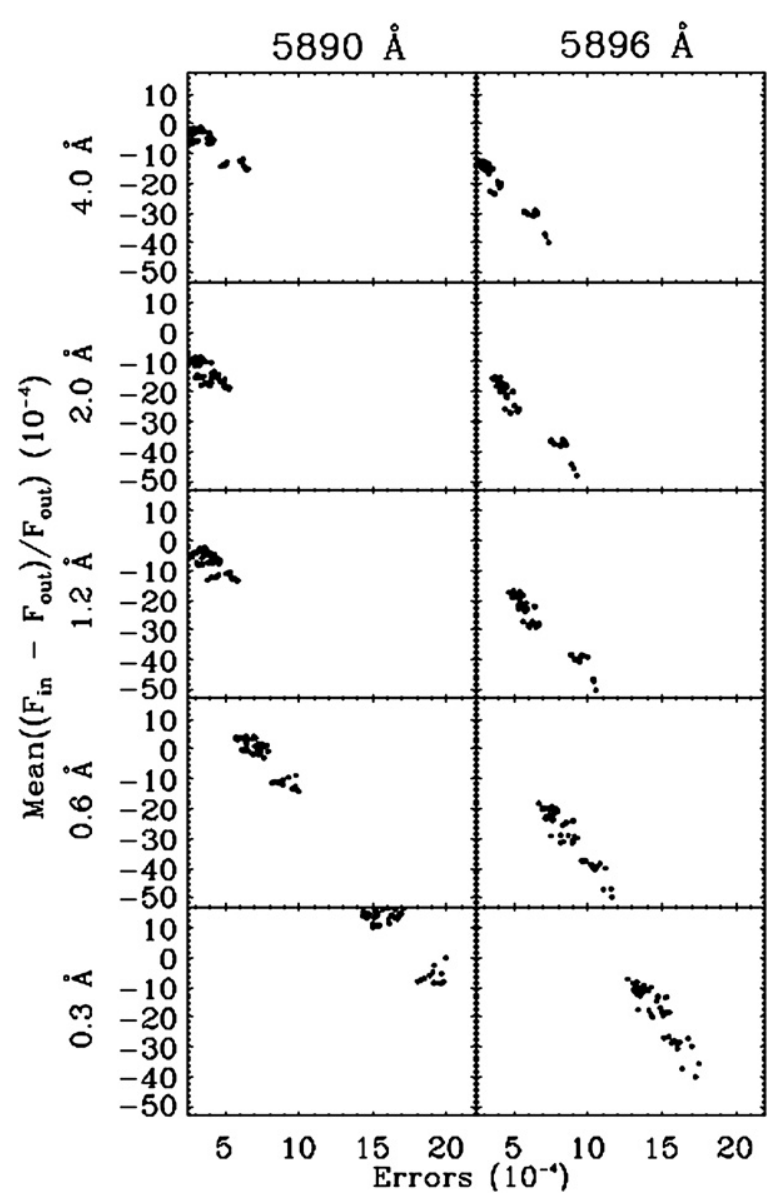

Figure 5. Monte Carlo results and uncertainties for each line of the $\mathrm{NaD}$ doublet. From top to bottom, Monte Carlo results for bands of 4.0, 2.0 (N2005), 1.2, 0.6, and $0.3(\mathrm{~N} 2005) \AA$. On the left, the $5890 \AA \mathrm{Na}$ line, on the right, the $5896 \AA$ line. The results quoted in Table 6 are the means of each of these distributions. The results and the uncertainties appear to be regularly distributed.

not viable for our data set because with smaller numbers of spectra, the statistical contribution to the uncertainty would rise rapidly.

Combining all the information from Figures 3 to 7, two distinct possible interpretations of the results emerge. First, if the transit is expected to produce no additional absorption in $\mathrm{Ni}$ I and Fe I, then the apparent detection of absorption in some of these other lines casts serious doubt on the detections of $\mathrm{NaD}$, and the data reduction and analysis procedures are suspect. For example, the Fe I 5906, 5910, and 5914 Å results getting more significant as more continuum is included implies that the continuum is not adequately normalized. However, if the Na results in Table 6 mean that the effective width of the $\mathrm{Na}$ absorption is no wider than $4 \AA$, then the wing contamination is minimal. A second possible interpretation is that the regularity of the distributions of the Monte Carlo results is just as significant as the strong results themselves, and is evidence that the measured $\mathrm{NaD}$ absorption is real, which implies that this work may have also detected absorption in $\mathrm{Ni}$ I, and probed the upper limit of absorption in Fe I.

There may be other reasons why additional absorption may appear in the $\mathrm{NaD}$ doublet, which are unrelated to transmission through a giant planet's atmosphere. C2002 discuss several alternate explanations for their detection. All of the alternate explanations are either not applicable to our method or were dismissed by $\mathrm{C} 2002$, as follows. 


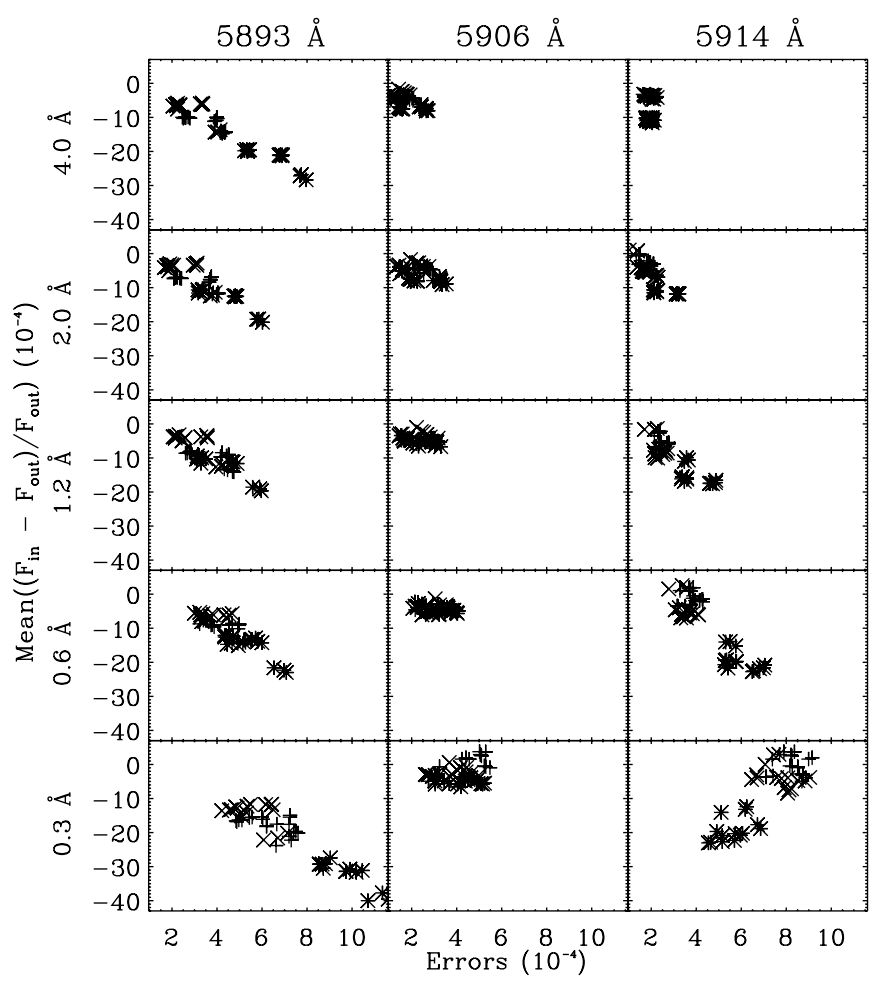

Figure 6. Monte Carlo results and uncertainties for other apparently marginal line detections. From top to bottom, Monte Carlo results for bands of 4.0, 2.0 (N2005), 1.2, 0.6, and 0.3 (N2005) A. From left to right, Monte Carlo results for the bands Ni I 5893, Fe I 5906, and Fe I $5914 \AA$. The results quoted in Table 6 are the means of each of these distributions. The symbols represent different terrestrial atmosphere removal processes-plusses: air-mass scaling HR 8634, stars: arbitrary scaling HR 8634, crosses: HITRAN models.

1. Wavelength-dependent stellar limb darkening. If stellar limb darkening has wavelength-dependent features on the order of $10 \AA$ in size, it may produce a false detection of $\mathrm{NaD}$ by straddling both of the doublet lines. Away from the $\mathrm{NaD}$ doublet, limb-darkening features $10 \AA$ in size are likely to vanish after the data have been continuum-normalized.

2. Limb darkening exhibited specifically by the sodium line relative to the continuum. C2002 generated model transit curves to test this and found this not to be a problem.

3. Nonlinearity in the CCD. Because the chip has a spectral coverage of $85 \AA$, and any minimum in a second (or higher) degree polynomial can mimic an absorption feature, the maximum size of any nonlinearity in the chip that may persist in the data after continuum-normalizing is approximately $10 \AA$. CCD nonlinearities larger than $10 \AA$ that survived flat-fielding will be removed by the continuumnormalizing process.

4. What if the star appears smaller at the sodium wavelengths, so the missing light is because the star radiates over less effective area at those wavelengths? C2002 estimated that the star would appear $10 \mathrm{~km}$ LARGER, and have the opposite, though negligible effect.

\section{CONCLUSIONS}

This work, obtained at higher resolution, detects $\approx 4.7$ times stronger in-transit absorption (in wavelength bins which encompass both lines of the $\mathrm{NaD}$ doublet) as $\mathrm{C} 2002$, with both results occurring at about the same $4 \sigma$ level of significance. Our results in the $4.0 \AA$ band are $\approx 1.6$ times smaller than the $4.4 \AA$ wide results reported by Sing et al. (2008) for HD 209458b. It has

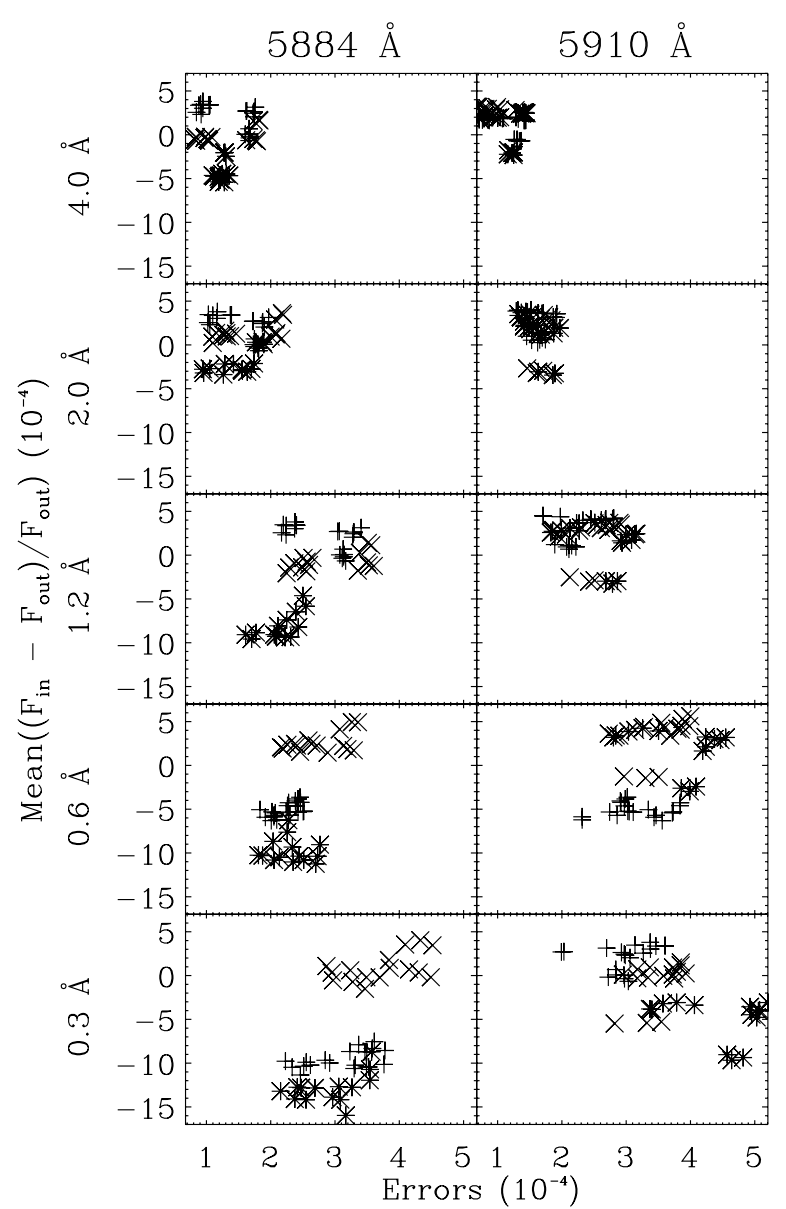

Figure 7. Monte Carlo results and uncertainties for other line nondetections. From top to bottom, Monte Carlo results for bands of 4.0, 2.0 (N2005), 1.2, 0.6 , and 0.3 (N2005) $\AA$. From left to right, Fe I 5884 and Fe I $5910 \AA$. The results quoted in Table 6 are the means of each of these distributions. For narrower wavelength bands, the results become more uncertain, and diverge to show either emission or absorption. The symbols represent different telluric line removal processes-plusses: air-mass scaling HR 8634, stars: arbitrary scaling HR 8634, and crosses: HITRAN models.

been pointed out (Seager 2003) that the C2002 detection was about a factor of 3 lower than expected. When combined with the results of S2008 and Sing et al. (2008), our results suggest that the atmosphere may be more variable than models predict, or alternately that accurate measurements of the atmosphere are still difficult. Also, loosening the LTE assumption in planetary atmosphere models results in the expected feature being deeper still (Barman et al. 2003).

Our results are also $\approx 1.6$ times larger than the "narrow" band results reported by Redfield et al. (2008) for HD 189733b. HD 209458b and HD 189733b are the two exoplanets most amenable to transmission spectroscopy of their atmospheres because their host stars have the brightest visual magnitudes $(v=$ 7.65-7.67) out of all the stars with planets thus far detected with transit photometry. Though the $\mathrm{C} 2002$ results suggest that these two planets would have very different Na properties based on mounting evidence of differences between their two atmospheres, ours do not. HD 209458b has a temperature inversion (Knutson et al. 2008) and their physical structures differ (i.e., HD 209458b has a larger-than-expected radius, HD 189733b is not). If the $\mathrm{C} 2002$ result is closer to the true value, then perhaps the $\mathrm{Na}$ is confined to lower layers in the atmosphere. If Sing et al. (2008) and our work are more representative to the true 
value, then perhaps the tidal heating has simply raised all cloud layers higher above the planet's core.

The present results share two characteristics with N2005. First, additional in-transit absorption is not consistent between both individual lines of the NaD doublet. N2005 marginally detect the $5890 \AA$ line using a $0.3 \AA$ A bandwidth, but do not detect the $5896 \AA$ line. We detect the $5896 \AA$ line, but only detect the 5890 Å line marginally at best. These seemingly opposite results for each individual line result from the difficult continuum normalization. Second, both N2005 and we obtain stronger intransit absorption signals than C2002. N2005 interpret this to mean that they do not have the same sensitivity as C2002. But there are other possible interpretations. The first is simply that the atmosphere of HD 209458 is variable and we are detecting different thicknesses or heights of the cloud cover than C2002. Second, C2002 show a smaller detection because the narrowest bandwidth they use in their lower-resolution STIS spectra does not completely resolve the $\mathrm{NaD}$ doublet, thus the $\mathrm{C} 2002$ result of $2.32 \times 10^{-4}$ includes a lot of continuum which should not show additional absorption. Resolutions of at least 30,000 are required to completely resolve all line species.

Both N2005 and this work have much higher spectral resolution, and are able to focus on the cores of the $\mathrm{NaD}$ lines, where one expects to see the strongest effect of the planet's atmosphere. With these high-resolution spectra, one should be able to indirectly measure the effective width of the planet's absorption signature, the smallest bandwidth with the best $\mathrm{S} / \mathrm{N}$. But further work is needed, both in improving the model atmospheric spectra used, and in testing the robustness of the results. Our results, combined with those of N2005, are only able to confirm the presence of the $\mathrm{C} 2002$ detection using ground-based telescopes after confronting difficult challenges.

The lessons learned from this work point to several suggestions for improvement on future ground-based attempts to detect extrasolar planet atmospheres from differential spectroscopy. First, as discussed in Section 3.3, the quantity of observations directly affects the statistical uncertainty, as one factor in the total photon statistics. While this work has the same photons per $\AA$ per spectrum as C2002, C2002 have a factor of 7 times more spectra, for a factor of 2.6 better expected uncertainty. Also, atmospheric water absorption is a very difficult noise source to content within the $\mathrm{NaD}$ region. Detailed modeling of terrestrial water features, combined with rapidly interleaved B-star spectra (obtained at similar air mass) offer the best hope of removing this noise source. One should probably increase the duty cycle spent on B stars to $50 \%$. Additionally, the unpredictable variation in the sensitive wavelength-dependent vignetting of most echelle spectrometers causes the continuum to vary by factors much larger than the size of the sought-after features. Better continuum-fitting techniques coupled with fiber-scrambling would both help in improving detectability.
This work was supported by grant NSF AST-0307493. We are grateful to Al Conrad of the W. M. Keck Observatory, for heroic work in restoring the computer system in time for the night's transit. The anonymous referee provided useful suggestions to improve the manuscript. We also owe respectful appreciation to our gracious hosts, the native people of Hawaii, whose allowance of our scientific visits to the Mauna Kea summit gives us the opportunity to explore the processes of nature.

\section{REFERENCES}

Barman, T. S., Hauschildt, P. H., Schweitzer, A., Stancil, P. C., Baron, E., \& Allard, F. 2003, in ASP Conf. Ser. 294, Scientific Frontiers in Research on Extrasolar Planets, ed. D. Deming \& S. Seager (San Francisco, CA: ASP), 467

Brown, T. M. 2001, ApJ, 553, 1006

Brown, T. M., Charbonneau, D., Gilliland, R. L., Noyes, R. W., \& Burrows, A. 2001, ApJ, 552, 699

Brown, T. M., Libbrecht, K. G., \& Charbonneau, D. 2002, PASP, 114, 826

Brown, T. M., et al. 2000, A\&AS, 197, 1105

Cameron, A. C. 2002, Astron. Geophys., 43, 21

Charbonneau, D. 2003, in ASP Conf. Ser. 294, Scientific Frontiers in Research on Extrasolar Planets, ed. D. Deming \& S. Seager (San Francisco, CA: ASP), 449

Charbonneau, D., Brown, T. M., Latham, D. W., \& Mayor, M. 2000, ApJ, 529, L45

Charbonneau, D., Brown, T. M., Noyes, R. W., \& Gilliland, R. L. 2002, ApJ, 568, 377 (C2002)

Charbonneau, D., Noyes, R. W., Korzennik, S. G., Nisenson, P., Jha, S., Vogt, S. S., \& Kibrick, R. I. 1999, ApJ, 522, L145

Davies, D. W. 1980, Icarus, 42, 145

Henry, G. W., Marcy, G. W., Butler, R. P., \& Vogt, S. S. 2000, ApJ, 529, L41

Hubbard, W. B., Fortney, J. J., Lunine, J. I., Burrows, A., Sudarsky, D., \& Pinto, P. 2001, ApJ, 560, 413

Knutson, H. A., Charbonneau, D., Allen, L. E., Burrows, A., \& Megeath, S. T. 2008, ApJ, 673, 526

Knutson, H. A., Charbonneau, D., Noyes, R. W., Brown, T. M., \& Gilliland, R. L. 2007, ApJ, 655, 564

Ksanfomality, L. V. 2004, Sol. Syst. Res., 38, 372

Lawrimore, J. H., et al. 2000, Bull. Am. Meteorol. Soc., 82, 1304

Levinson, D. H., \& Waple, A. M. 2003, Bull. Am. Meteorol. Soc., 85, 811

Marcy, G. W., \& Butler, R. P. 1998, ARA\&A, 36, 57

Mayor, M., \& Queloz, D. 1995, Nature, 378, 355

Mazeh, T., et al. 2000, ApJ, 532, L55

Narita, N., et al. 2005, PASJ, 57, 471 (N2005)

Redfield, S., Endl, M., Cochran, W. D., \& Koesterke, L. 2008, ApJ, 673, L87

Seager, S. 2003, in ASP Conf. Ser. 294, Scientific Frontiers in Research on Extrasolar Planets, ed. D. Deming \& S. Seager (San Francisco, CA: ASP), 457

Seager, S., \& Sasselov, D. D. 2000, ApJ, 537, 916

Sing, D. K., Vidal-Madjar, A., Desert, J.-M., Lecavelier des Etangs, A., \& Ballester, G. 2008, arXiv:0802.3864

Snellen, I. A. G., Albrecht, S., de Mooij, E. J. W., \& Le Poole, R. S. 2008, A\&A, 487, 357

Vidal-Madjar, A., Lecavelier des Etangs, A., Désert, J.-M., Ballester, G. E., Ferlet, R., Hébrard, G., \& Mayor, M. 2003, Nature, 422, 143

Vidal-Madjar, A., et al. 2004, ApJ, 604, L69

Vogt, S. S., et al. 1994, Proc. SPIE, 2198, 362

Welsh, B. Y., Crifo, F., \& Lallement, R. 1998, A\&A, 333, 101

Welsh, B. Y., Vedder, P. W., Vallerga, J. V., \& Craig, N. 1991, ApJ, 381, 462 